\title{
Behaviour of magnetotelluric source fields within the equatorial zone
}

\author{
Antonio L. Padilha \\ Instituto Nacional de Pesquisas Espaciais - INPE, C.P. 515, 12201-970 São José dos Campos, Brazil
}

(Received October 10, 1998; Revised May 4, 1999; Accepted May 4, 1999)

\begin{abstract}
It is well known that equatorial electrojet (EEJ) currents can significantly affect the geomagnetic variations. However, in a recent study (Padilha et al., 1997) it was observed that magnetotelluric (MT) soundings carried out across the dip equator in the Brazilian equatorial zone were not affected significantly due to EEJ currents. By using new results from geomagnetic variation signals, measured simultaneously to the MT experiment at a chain of equatorial and mid-latitude stations, an attempt is made here to explain the MT results in terms of the behaviour of the primary inducing field during the survey. Most of the analysis is performed by considering a single frequency $(0.885 \mathrm{mHz})$, representative of the MT frequency interval. It is observed that the amplitude of the geomagnetic variations appears horizontally homogeneous within the study area (from $-3^{\circ}$ to $+3^{\circ}$ of geomagnetic latitude), indicating that the primary field in the analysed frequency range may be considered sufficiently uniform in the horizontal direction thus satisfying the Tikhonov-Cagniard plane-wave criterion. The same geomagnetic data also show that, if any EEJ source effect exists, it would be restricted to the transition zone (between $3^{\circ}$ and $5^{\circ}$, at both sides of the dip equator). Dmitriev-Berdichevsky's constraints calculated at two different frequencies and a modelling exercise using EEJ parameters derived from a magnetometer array were able to explain the MT observations and have shown that source effects would just appear in frequencies lower than $1 \mathrm{mHz}$ (resistive regions) and $0.1 \mathrm{mHz}$ (conductive regions). Considering the characteristics of propagation and amplification of geomagnetic variations at the equatorial zone it is concluded that EEJ currents could be used as a source for lithospheric MT studies in these regions.
\end{abstract}

\section{Introduction}

In magnetotelluric (MT) studies, the primary electromagnetic inducing field originating from the ionosphere and magnetosphere is assumed to be laterally sufficiently uniform over the study region and it is usually described as plane waves (Tikhonov, 1950; Cagniard, 1953). The validity of such a basic assumption is questionable at auroral and equatorial latitudes where ionospheric electrojet currents are present and can make the primary field nonuniform (the so-called source effects).

A number of theoretical studies (e.g., Mareschal, 1986; Pirjola, 1992; and references therein) have discussed possible source effects at auroral and equatorial regions and have demonstrated that when the nonuniformity of the sources is considered the Tikhonov-Cagniard formula may require modification to compute the Earth's transfer functions at frequencies lower than $0.01 \mathrm{~Hz}$ (periods larger than $100 \mathrm{~s}$ ). However, in a previous paper Padilha et al. (1997), herein after called as PAPER1, have discussed a broad band MT survey carried out across the magnetic dip equator at the Brazilian equatorial zone and have shown that the equatorial electrojet (EEJ) currents do not significantly affect the MT soundings. These results can be seen as an assurance that the traditional plane wave formula can provide reliable subsurface conductivity structure even under the influence

Copy right (C) The Society of Geomagnetism and Earth, Planetary and Space Sciences (SGEPSS); The Seismological Society of Japan; The Volcanological Society of Japan; The Geodetic Society of Japan; The Japanese Society for Planetary Sciences. of the EEJ, at least up to the lowest frequency measured (0.488 mHz; period of $2048 \mathrm{~s}$ ).

In the present study an attempt has been made to explain the MT results in terms of the behaviour of the primary field during the survey. The broad nature of latitudinal variation of the source fields in the equatorial region of Brazil is analysed using new results from geomagnetic variation signals measured at a chain of ground-based temporary and permanent stations operating simultaneously with the MT experiment. Also, the theoretical estimation of the EEJ source effects on the acquired MT data is recalculated considering ionospheric parameters derived from ground-based geomagnetic measurements and a discussion is made based on the possible use of EEJ currents as the pumping field for lithospheric induction studies.

\section{Geomagnetic Data}

The magnetometer data used here were obtained from four temporary stations (POV, ARI, PRM, and VIL), operated at the same region and simultaneously to the MT survey, and two longitudinally separated near-equatorial (EUS) and midlatitude (VAS) permanent observatories. Figure 1 shows the location of the stations. Three-axial fluxgate magnetometers were used but the instrument at VAS (a linear sensor; Trigg et al., 1971) is different from those at the other stations (ring core sensor; Acuña, 1974). The sampling time and amplitude resolution were respectively $3 \mathrm{~s}$ and $0.015 \mathrm{nT}$ for the ring core and $60 \mathrm{~s}$ and $0.1 \mathrm{nT}$ for the linear sensor. For compatibility, all the data will be presented with a sampling rate of $60 \mathrm{~s}$ and 


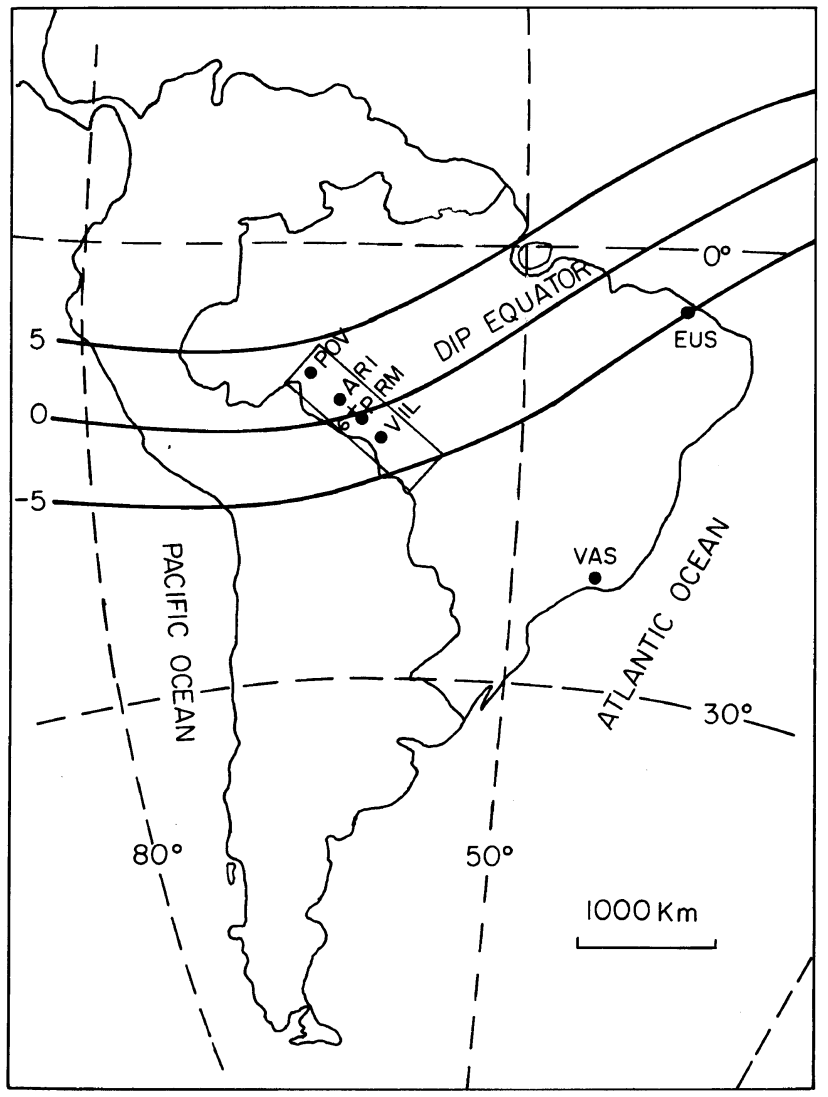

Fig. 1. Map of South America showing the location of the geomagnetic (POV, ARI, PRM, VIL, EUS, and VAS) and MT (cross for number 6) stations referred to in this work (MT station 5 is not shown because it is geographically coincident with geomagnetic station PRM). Inset is the region of the MT survey (figure 1 in PAPER1). Also shown are the calculated isolines of the geomagnetic latitude (based on the IGRF-1995 coefficients)

a resolution of $0.1 \mathrm{nT}$.

To exemplify the behaviour of the geomagnetic variations at the Brazilian equatorial region during the MT fieldwork, a 5-day period was chosen (between 16 and 20 of October, 1995) when the two MT stations closest to the dip equator were recorded (stations 5 and 6 in PAPER1). Also, this period encompasses two geomagnetically quiet days (16 and 17 ) and three disturbed days (18 to 20$)$, including a magnetic storm with sudden commencement at 11:22 UT on October, 18. A target frequency $(0.885 \mathrm{mHz}$; period of $1130 \mathrm{~s})$ was selected at the lower end of the frequency range of the MT data (expected to have larger EEJ effects) and the geomagnetic data were band-pass filtered using a zero-phase shift Butterworth function (Kanasewich, 1981) with unit response between 0.771 and $1.026 \mathrm{mHz}$, corresponding to the bandwidth where band averaging is performed to calculate the MT transfer functions at the target frequency.

Figure 2 shows a comparison between filtered geomagnetic variations during the chosen 5-day period and hourly variation of recorded scalar MT transfer functions at the target frequency in sites 5 and 6 . Geomagnetic signals are represented by filtered $\mathrm{B}_{x}$ (geomagnetic north-south) component (expected to be the most affected by the diurnal eastwest EEJ currents) at the stations PRM and VAS, respec- tively inside and outside the EEJ influence. In this graph, the above referred sudden commencement occurs at $59 \mathrm{~h}$ $22 \mathrm{~m}$. The MT transfer functions were calculated for the east-directed electric field $\left(\mathrm{E}_{y}\right)$ and north-directed magnetic field $\left(\mathrm{B}_{x}\right)$. Non-overlapping 1-hour data-length segments were used to obtain the hourly variation of apparent resistivity and impedance phase responses at the target frequency for the two MT stations.

The already known effect of the EEJ amplifying the daytime geomagnetic oscillations at the equatorial station (PRM) is highlighted in the figure. On the other hand, in spite of the reduced number of points used to calculate the Fourier coefficients in the chosen frequency (a little more than 3 periods), at both MT stations the hourly transfer functions exhibit consistent results during most of the data acquisition interval (including the period of the previously referred geomagnetic storm). It is not observed any daytime effect that could be associated with the EEJ or any other significant short-period oscillations of the EEJ currents (time scales of half an hour to a few hours), known to occur on some given days (Forbes, 1981; Reddy, 1989). On the contrary, the largest fluctuations in the MT data are observed in nighttime, during low geomagnetic activity (from 46 to $58 \mathrm{~h}$ and from 71 to $82 \mathrm{~h}$ ). These observations confirm that the signal level is a very important factor in MT surveys and indicate that the best period to acquire high-quality MT data in equatorial regions is during daytime.

\section{Latitudinal Variation}

The horizontal variation of the geomagnetic oscillations at the chosen frequency in the north-south direction (called here as latitudinal variation) was obtained by comparing the amplitude variation of the filtered signal at the six geomagnetic stations. Figure 3 shows the stacked plots of the filtered data in the $\mathrm{B}_{x}$-component for the interval of 38-42 h, corresponding to 10-14 LT on October 17, 1995. This interval was chosen because at the analysed frequency the maximum amplitude enhancement due to EEJ currents is expected to occur around noon hours (Sarma and Sastry, 1995).

Two groups of stations can be seen from this picture: two stations with smaller amplitudes (VAS and EUS, located outside and at the border of the EEJ influential area, respectively) and four stations with larger amplitudes (POV, ARI, PRM and VIL, the stations close to the dip equator). In this second group, one station presents slightly smaller amplitude than the others (POV, inside the EEJ area but more distant from the dip equator). Mean amplitude variations are presented in Table 1. The differences in the mean amplitude between the three central stations are very small (ratio of 1.1 between the mean amplitude of PRM and VIL), indicating that the signal can be considered nearly homogeneous around the center of the EEJ (from ARI to VIL).

The power characteristics of the filtered signals were obtained through an FFT-based analysis. The dynamic variation of power estimates at each station during the 5-day period was combined in Fig. 4, giving a three-dimensional plot of the power variation as a function of time and geomagnetic latitude (using the geomagnetic coordinates of Table 1).

Again the daytime amplification of the geomagnetic variations at the equatorial zone is clearly seen (as in the case of the 

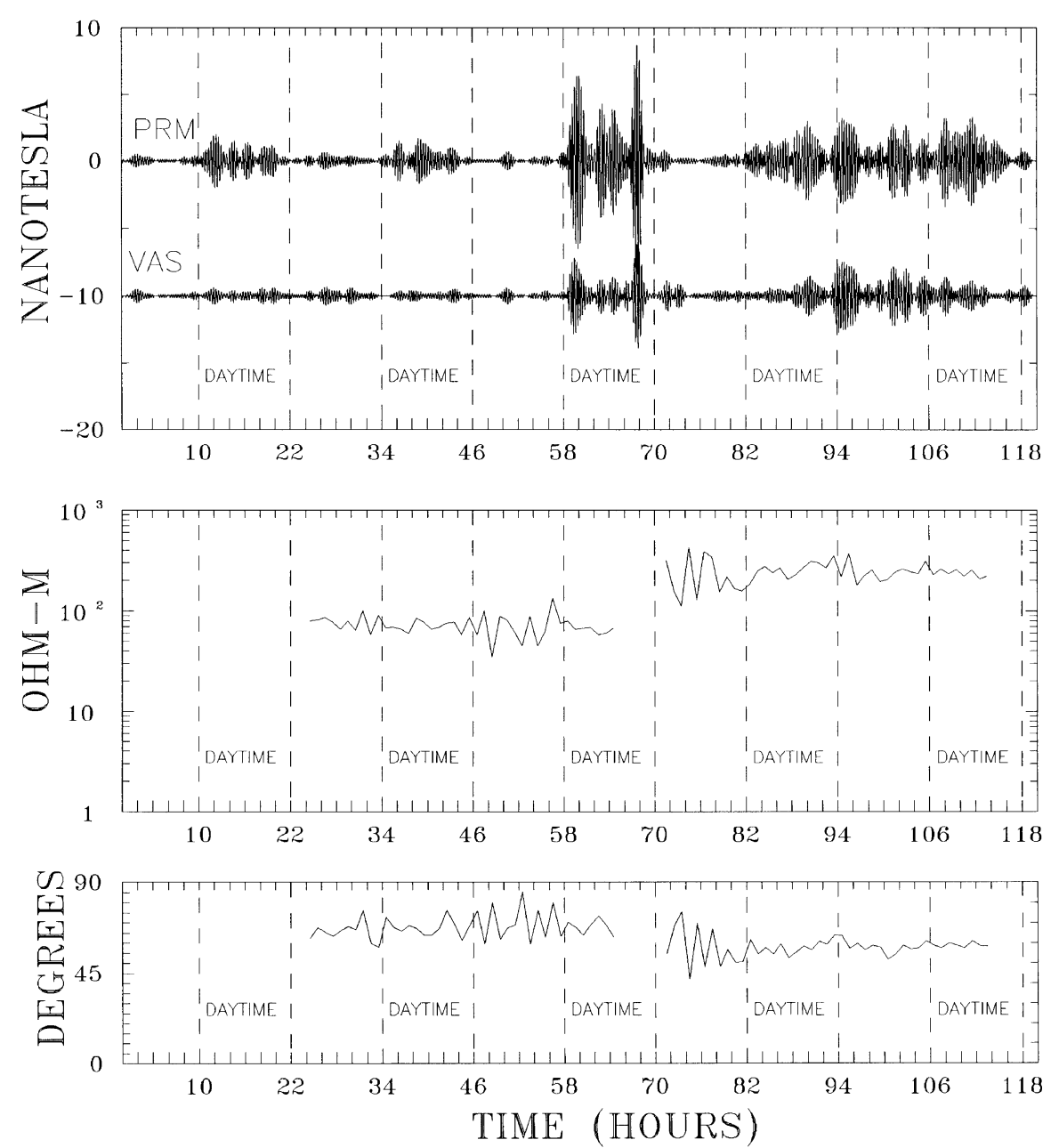

Fig. 2. Comparison of geomagnetic variations and recorded MT transfer functions in the period between 16th and 20th of October, 1995. Upper panel: amplitude of band-pass $(0.771-1.026 \mathrm{mHz})$ Butterworth filtered $\mathrm{B}_{x}$-component magnetic variations at stations PRM and VAS; intermediate panel: hourly variation of apparent resistivity at the frequency $0.885 \mathrm{mHz}$ for the east-directed electric field at sites 5 (24 to $67 \mathrm{~h})$ and 6 ( $71 \mathrm{ho} 114 \mathrm{~h}$ ); lower panel: the same as the intermediate panel for the phase response.

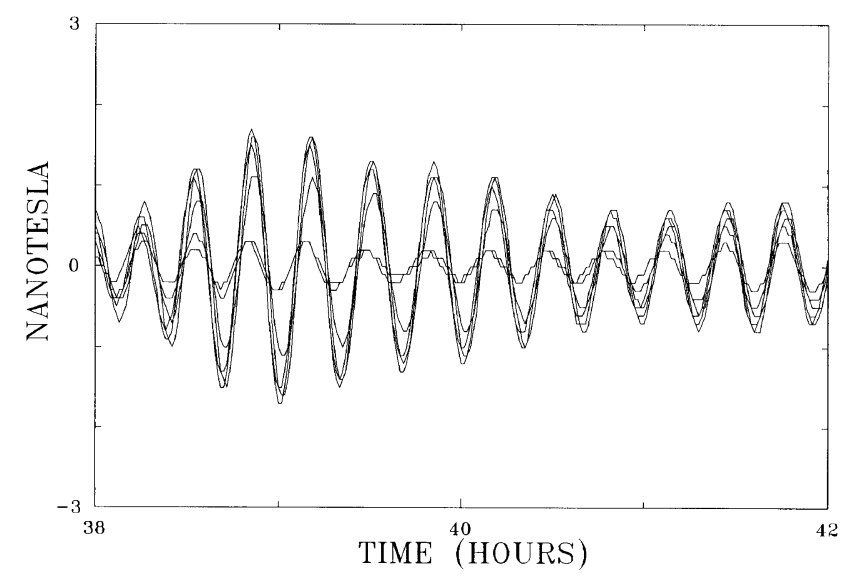

Fig. 3. Stacked plots of band pass Butterworth filtered geomagnetic variations in the $\mathrm{B}_{x}$-component for the time interval of 38 to $42 \mathrm{~h}$ in Fig. 2 (10-14 LT on October 17, 1995).

filtered data of Fig. 2). No sharp spatial variation of power is detected under the dip equator, with the amplitude of the geomagnetic variations appearing horizontally homogeneous
Table 1. Mean amplitude of the band-pass filtered $(0.771$ to $1.026 \mathrm{mHz})$ geomagnetic variations shown in Fig. 3 (values in parentheses are one standard deviation).

\begin{tabular}{ccc}
\hline Station & $\begin{array}{c}\text { Geomagnetic } \\
\text { latitude }\left({ }^{\circ}\right)\end{array}$ & Amplitude (nT) \\
\hline POV & 2.9 & $1.5(0.4)$ \\
ARI & 1.6 & $2.2(0.6)$ \\
PRM & -0.1 & $2.2(0.7)$ \\
VIL & -1.9 & $2.0(0.7)$ \\
EUS & -4.7 & $0.5(0.2)$ \\
VAS & -17.7 & $0.5(0.2)$ \\
\hline
\end{tabular}

within roughly $\pm 3^{\circ}$ of latitude. A shift towards the north of the electrojet axis is observed between 58 and $70 \mathrm{~h}$ (daytime of the disturbed day 18th of October), normally associated with neutral wind effects (Fambitakoye and Mayaud, 1976; Reddy, 1989). During this day the maximum difference in 


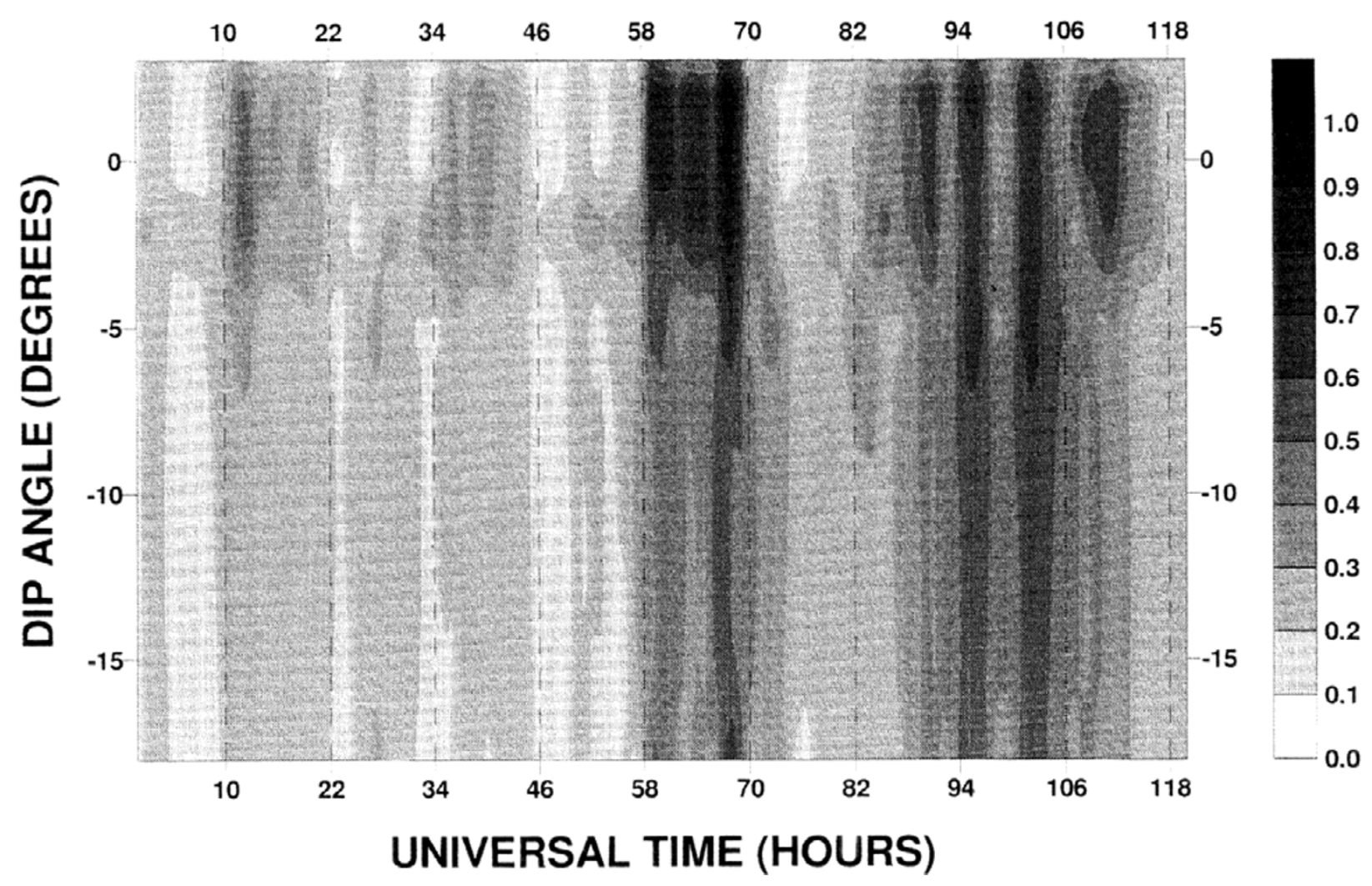

Fig. 4. Relative variation of power at the frequency of $0.885 \mathrm{mHz}$ as a function of time and latitude in the period from 16 th to 20 th of October, 1995 .
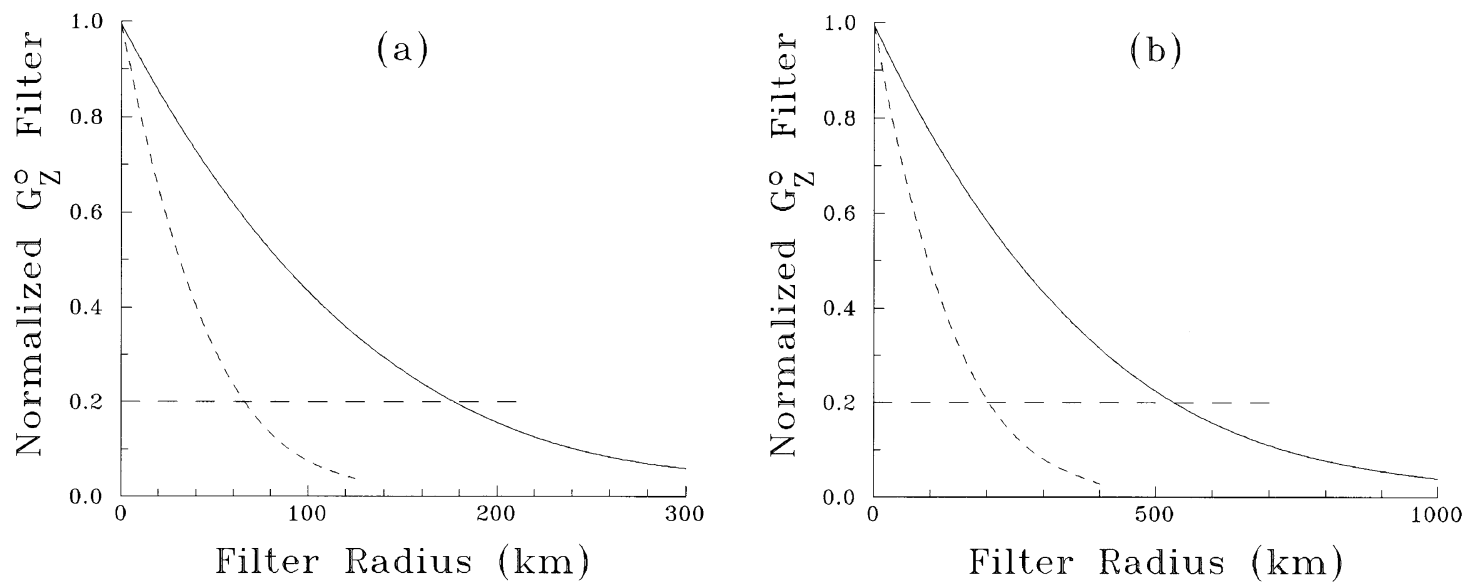

Fig. 5. Effective characteristics of the $G_{Z}^{\circ}$ filter for one-dimensional conductive (dashed lines) and resistive (continuous line) models for site 6. (a) frequency of $0.885 \mathrm{mHz}$; (b) frequency of $0.1 \mathrm{mHz}$.

amplitude between the stations PRM and VIL (ratio of 1.3) is observed, but not affecting the MT results at site 5 (see the MT transfer functions between 58 and $62 \mathrm{~h}$ in Fig. 2).

It is known that the application of the plane wave MT equation is conditioned by the spatial dependence of the primary field. Dmitriev and Berdichevsky (1979) showed that if, at the surface of a layered earth, the horizontal magnetic field varies linearly over distances of about three skin depths then the Tikhonov-Cagniard formula is usable. Their technique of determining the region over which the field constraints must be respected was applied to the one-dimensional models for site 6 (PAPER1). The Dmitriev-Berdichevsky filter $G_{Z}^{\circ}$, normalized by the factor $1 / \omega \mu_{\circ}$, is calculated as a function of the radial distance $(r)$ using the equation:

$$
\left|\frac{G_{Z}^{\circ}}{\omega \mu_{\circ}}\right|=\mathrm{e}^{-r / d}
$$

where $\omega$ is the frequency, $\mu_{\circ}$ the magnetic permeability and $d$ the skin depth.

Figure 5 shows the effective characteristics of the normalized filters, calculated at two different frequencies for the 
two models discussed in PAPER1 for site 6. A limit of 0.2 is imposed to the normalized filter values to obtain the size of the area where the Tikhonov-Cagniard formula can be applied (Dmitriev and Berdichevsky, 1979). For the frequency of $0.885 \mathrm{mHz}$ (left side of the figure), it can be seen that the filter radius roughly corresponds to $65 \mathrm{~km}$ (conductive model) and $175 \mathrm{~km}$ (resistive model). Consequently, the diameter of the region surrounding the station within which the Dmitriev-Berdichevsky's constraints are valid is found to be $130 \mathrm{~km}$ (conductive model) and $350 \mathrm{~km}$ (resistive model). As the present study has shown that the horizontal field changes in the Brazilian equatorial zone are smooth and gradual over distances of at least $400 \mathrm{~km}$ (ARI to VIL), it is expected that EEJ source effects would not affect significantly the MT data at site 6 at that frequency.

As an additional exercise, the same procedure was performed considering a lower target frequency $(0.1 \mathrm{mHz}$; period of $10,000 \mathrm{~s})$. Slightly sharper variations under the dip equator (ratio of 1.3 between PRM and VIL in the same time interval of Fig. 3) are observed, but the general characteristics of the dynamic power variation are not different from the frequency of $0.885 \mathrm{mHz}$ presented in Fig. 4 (roughly horizontally homogeneous within $\pm 3^{\circ}$ of latitude). However, it can be seen in Fig. 5 (right side) that the diameter of the region over which the Dmitriev-Berdichevsky's conditions must prevail is enlarged to $400 \mathrm{~km}$ (conductive model) and $1050 \mathrm{~km}$ (resistive model). Consequently, the source effects are probably more prominent at that frequency, specially for the resistive model.

\section{Modelling Electrojet Effects}

As discussed in PAPER1, an estimation of the possible EEJ source effects on the diurnal MT data was carried out considering two different models (line-current and Gaussian) to represent the EEJ sources. It was observed that the linecurrent model generated departures from the plane-wave results not observed in practice and that the Gaussian model only agreed with the experimental data, within their error bars, if the geoelectric section could be represented by the most conductive model.

In that paper, the EEJ sources were represented by parameters derived from the POGO satellites scalar data (Cain and Sweeney, 1972). However, the MAGSAT data (Langel et al., 1982) evidenced that ionospheric field variations should, presumably, be better reduced using vector data since their effect on each component can be observed separately and checked for consistency with existing models of ionospheric sources (Cohen and Achache, 1990). The main consequence of using the POGO data to derive the EEJ parameters is that the calculated halfwidth is consistently shorter than observations (Onwumechili and Ezema, 1992).

In order to define the EEJ parameters consistent with the ground observations at the Brazilian equatorial zone, a numerical simulation using the Gaussian model has been performed for different halfwidths. It is observed that in order to obtain the horizontal field changes as shown in Fig. 4, the EEJ halfwidth would have to be larger than $400 \mathrm{~km}$.

More quantitative information on the morphology of the EEJ can be obtained through more detailed studies. In a recent paper, Rigoti et al. (1999) describe the results of an
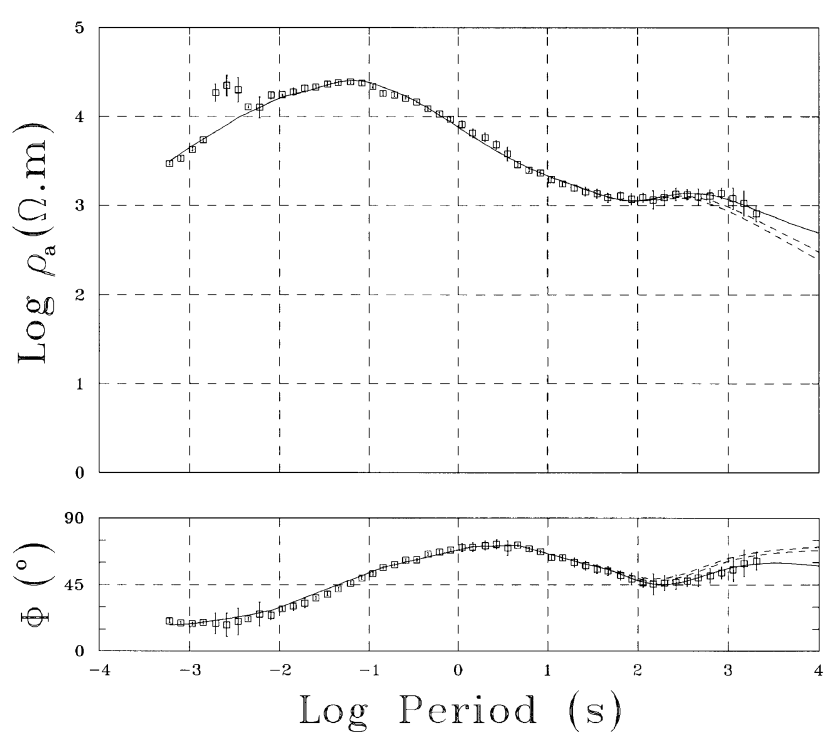

Fig. 6. Comparison of daytime rotationally invariant MT data at site 6 with curves associated with different fields incident on the resistive model of PAPER1. Continuous line is the plane wave field and the dashed lines represent the two extreme Gaussian electrojet fields given by the Gaussian upper and lower halfwidth limits (see text for explanation). Error bars are one standard deviation.

array of 29 vector magnetometers operated during 4 months in N-NE Brazil. EEJ's main parameters averaged for 16 quiet days were $403 \pm 67 \mathrm{~km}$ for the halfwidth and $67 \pm 20\left(10^{3} \mathrm{~A}\right)$ for the total eastward current. These results are compared well with the EEJ halfwidth (350 to $400 \mathrm{~km}$ ) and current intensities (20,000 to 70,000 A) found by Hesse (1982) from a previous equatorial profile study around the same longitude in Brazil.

The possible EEJ source effects on the daytime MT data of site 6 were then recalculated using the Rigoti et al. (1999) parameters to represent the EEJ currents. In Fig. 6, a comparison is made between the experimental data (apparent resistivities and phases) and the theoretical ones derived from both plane-wave and Gaussian electrojet fields incident on the resistive model of PAPER1 (the most critical in the modelling carried out at that paper). Conservatively, two different halfwidths were used in the Gaussian models, given by the lower $(336 \mathrm{~km})$ and upper $(470 \mathrm{~km})$ limits of the standard error. Two extreme curves are then generated, within which the experimental data would have to be present.

It is observed that the difference between the two curves obtained using the extreme Gaussian models are quite small and the source effects (difference between the plane-wave and the Gaussian curves) start to distort the soundings for periods approaching $1000 \mathrm{~s}$ (frequencies of $1 \mathrm{mHz}$ ). Both the plane-wave and the Gaussian models agree with the experimental data within the error limit. The source effects only become more important for periods approaching 10,000 s (frequencies of $0.1 \mathrm{mHz}$ ), but there are no experimental data to confirm these results. Similar calculations on the conductive model of PAPER1 showed that the source effects would start at periods around $10,000 \mathrm{~s}$ (frequencies of $0.1 \mathrm{mHz}$ ) and would become significant for periods as large as 100,000 s (frequencies around $0.01 \mathrm{mHz}$ ). All these results are in 
close agreement with the ones derived from the DmitrievBerdichevsky's constraints.

\section{Discussion and Conclusions}

The present study shows that the horizontal geomagnetic field variations in the Brazilian equatorial zone during a previous MT survey were smooth and gradual over distances of at least $400 \mathrm{~km}$ (between stations ARI and VIL). When compared to the source behaviour at auroral regions, where the current systems are able to develop in surges on horizontal scales as small as a hundred kilometers (Mareschal, 1986), the field under the EEJ influence seems to behave in a much more constrained manner. In fact, the source field in the Brazilian equatorial zone obeys inclusive the stringent WaitPrice criterion of slowness of the geomagnetic field variations for applicability of the MT method (Wait, 1954; Price, 1962). The MT results can then be explained by assuring that source effects did not affect the data during the survey. Obviously, these conclusions are valid in the interval of $\pm 3^{\circ}$ of geomagnetic latitude, where the MT sites were located, and for signals in the 0.488 to $1000 \mathrm{~Hz}$ frequency range (periods from 0.001 to $2048 \mathrm{~s}$ ). Outside this region (specially in the zone between $3-5^{\circ}$, on both sides of the dip equator), the horizontal variation of the field is more pronounced (ratio of 3.0 in Table 1) and source effects may occur. In lower frequencies (closer to $0.1 \mathrm{mHz}$ ) the EEJ effects are probably present within the $\pm 3^{\circ}$ zone. Unfortunately there are no MT data available to verify these possibilities.

The geomagnetic data was also used to study the ionospheric current models at the equatorial region. It was observed that the previously used satellite-derived parameters were not adequate to depict the halfwidth of a Gaussian representation of the EEJ. The use of more realistic quantitative parameters, defined from a ground-based array study is able to explain the observations. The results of the theoretical modelling (Fig. 6) are also in close agreement to the ones derived from the Dmitriev-Berdichevsky's constraints of applicability of the traditional MT approximation (Fig. 5). These observations permit us to conclude that in resistive regions there is no source effect associated with the EEJ for frequencies higher than $1 \mathrm{mHz}$ (periods lower than $1000 \mathrm{~s}$ ), whereas over conductive regions the critical frequency seems to be $0.1 \mathrm{mHz}$ (period of $10,000 \mathrm{~s}$ ).

The potential of the strong EEJ currents in equatorial regions as a well defined source for earth conductivity studies can also be evaluated using the available data. Understanding the behaviour of the geomagnetic variations at equatorial latitudes in the frequency range of interest and, more specifically, on how the energy from the outer magnetosphere is coupled or propagates down to low latitudes are some of the necessary steps for such a research. Theoretical models (e.g., Kikuchi and Araki, 1979a,b) have shown that in the equatorial E-region there is a screening effect on the compressional hydromagnetic waves transmitted from the magnetosphere to the equatorial ionosphere. The main consequence is the decrease in amplitude of the magnetic variations near the dip equator during nighttime. In daytime, however, there is a local amplitude enhancement of the pulsations due to an instantaneous transmission of polar ionospheric electric field variations into the equatorial ionosphere and a local amplification of the signals at dip equator associated with much higher ionospheric conductivity. Recent experimental investigations on continuous (Pc5; Trivedi et al., 1997) and irregular (Pi2; Shinohara et al., 1997) pulsations give support to these models.

Considering these observations and the results from this paper, it can be stated that EEJ currents can be used as a forcing field for MT surveys in the equatorial region. To avoid source effects for lithospheric studies, the frequency range must be limited to the critical frequencies of $1 \mathrm{mHz}$ (over resistive regions) and $0.1 \mathrm{mHz}$ (in conductive environments). Also, due to the screening effect which affects mostly the nighttime recordings, the characteristics of daytime transmission and the amplification of daytime pulsation amplitude, it is recommended to acquire MT data during daytime only.

Acknowledgments. This study was supported by grants from FAPESP (proc. 93/0652-2 and 98/13825-6). I thank Í. Vitorello, N. B. Trivedi, B. R. Arora, and L. Rijo, for their important contributions to the field experiment and for valuable discussions during the course of this work, and T. Harinarayana and one anonymous reviewer, for helpful suggestions.

\section{References}

Acuña, M. H., Fluxgate magnetometers for outer planets exploration, IEEE T. Mag., 10, 519-523, 1974.

Cagniard, L., Basic theory of the magneto-telluric method of geophysical prospecting, Geophysics, 18, 605-635, 1953.

Cain, J. C. and R. E. Sweeney, The POGO data, J. Atmos. Terr. Phys., 35, 1231-1247, 1972

Cohen, Y. and J. Achache, New global vector magnetic anomaly maps derived from Magsat data, J. Geophys. Res., 95, 10783-10800, 1990.

Dmitriev, V. I. and M. N. Berdichevsky, The fundamental model of magnetotelluric sounding, Proc. IEEE, 67, 1034-1044, 1979.

Fambitakoye, O. and P. N. Mayaud, Equatorial electrojet and regular daily variation $\mathrm{S}_{R}$, II, The center of the equatorial electrojet, J. Atmos. Terr. Phys., 38, 19-26, 1976.

Forbes, J. M., The equatorial electrojet, Rev. Geophys., 19, 469-504, 1981. Hesse, D., An investigation of the equatorial electrojet by means of groundbased magnetic measurements in Brazil, Ann. Geophys., 38, 315-320, 1982.

Kanasewich, E. R., Time Sequence Analysis in Geophysics, pp. 237-280, The University of Alberta Press, Edmonton, Canada, 1981

Kikuchi, T. and T. Araki, Transient response of uniform ionosphere and preliminary reverse impulse of geomagnetic storm sudden commencement, J. Atmos. Terr. Phys., 41, 917-925, 1979a.

Kikuchi, T. and T. Araki, Horizontal transmission of the polar electric field to the equator, J. Atmos. Terr. Phys., 41, 927-936, 1979b.

Langel, R., G. Ousley, J. Berbert, J. Murphy, and M. Settle, The Magsat mission, Geophys. Res. Lett., 9, 243-245, 1982.

Mareschal, M., Modeling of natural sources of magnetospheric origin in the interpretation of regional induction studies: A review, Surv. Geophys., 8 261-300, 1986.

Onwumechili, C. A. and P. O. Ezema, Latitudinal and vertical parameters of the equatorial electrojet from an autonomous data set, J. Atmos. Terr. Phys., 54, 1535-1544, 1992.

Padilha, A. L., Í. Vitorello, and L. Rijo, Effects of the equatorial electrojet on magnetotelluric surveys: Field results from northwest Brazil, Geophys. Res. Lett., 24, 89-92, 1997.

Pirjola, R., On magnetotelluric source effects caused by an auroral electrojet system, Radio Sci., 27, 463-468, 1992.

Price, A. T., The theory of magnetotelluric methods when the source field is considered, J. Geophys. Res., 67, 1907-1918, 1962.

Reddy, C. A., The equatorial electrojet, Pure Appl. Geophys., 131, 485-508, 1989.

Rigoti, A., F. H. Chamalaun, N. B. Trivedi, and A. L. Padilha, Characteristics of the Equatorial Electrojet determined from an array of magnetometer in N-NE Brazil, Earth Planets Space, 51, 115-128, 1999.

Sarma, S. V. S. and T. S. Sastry, On the equatorial electrojet influence on 
geomagnetic pulsation amplitudes, J. Atmos. Terr. Phys., 57, 749-754, 1995.

Shinohara, M., K. Yumoto, A. Yoshikawa, O. Saka, S. I. Solovyev, E. F. Vershinin, N. B. Trivedi, J. M. Da Costa, and The $210^{\circ}$ MM Magnetic Observation Group, Wave characteristics of daytime and nighttime Pi 2 pulsations at the equatorial and low latitudes, Geophys. Res. Lett., 24, 2279-2282, 1997.

Tikhonov, A. N., On determining electrical characteristics of the deep layers of the Earth's crust, Dokl. Akad. Nauk. SSSR, 73, 295-297, 1950.

Trigg, D. F., P. H. Serson, and P. A. Camfield, A solid state electrical recording magnetometer, Publ. Dep. Energy Mines and Resources, Earth Phys.
Branch, 41, 67-80, 1971.

Trivedi, N. B., B. R. Arora, A. L. Padilha, J. M. Da Costa, S. L. G. Dutra, F. H. Chamalaun, and A. Rigoti, Global Pc5 geomagnetic pulsations of March 24, 1991 as observed along the American sector, Geophys. Res. Lett., 24, 1683-1686, 1997.

Wait, J. R., On the relation between telluric currents and the earth's magnetic field, Geophysics, 19, 281-289, 1954.

A. L. Padilha (e-mail: padilha@dge.inpe.br) 